\title{
Logic, Language, Information and Computation
}

\section{Preface}

This volume contains the peer-reviewed full versions of selected contributions presented at the 16th Workshop on Logic, Language, Information and Computation (WoLLIC 2009), held from June 21 through 24, 2009, in the National Center of Sciences, Tokyo, Japan.

WoLLIC is a series of workshops which started in 1994 with the aim of fostering interdisciplinary research in pure and applied logic. The idea of the workshop is to have a forum which is large enough in the number of possible interactions between logic and the sciences related to information and computation, and yet is small enough to allow for concrete and useful interaction across logic-related disciplines.

Held over the course of four full days, WoLLIC 2009 included both extended tutorial sessions and general lectures, given by an international panel of the world's top experts in the fields of theoretical and applied logic, linguistics, and computer science. The composition of the speakers and the other participants of the workshop reflected the diversity of the event: included were members of psycholinguistics, cognitive science, mathematics, philosophy, theoretical computer science and business software communities from geographical locales as close to home as Tokyo, to as far away places such as Brazil and Iran. The close contact between the speakers and the other participants ensued, within the intensive workshop environment, an intimate setting in which the free and fruitful exchange of ideas could take place.

The members of the Program Committee included: Toshiyasu Arai (Kobe University), Matthias Baaz (Vienna University of Technology), Alexandru Baltag (Oxford University), Josep Maria Font (University of Barcelona), Silvio Ghilardi (University of Milan), Katsumi Inoue (National Institute of Informatics), Marcus Kracht (University of Bielefeld), Hiroakira Ono (Japan Advanced Institute of Science and Technology) (Chair), Masanao Ozawa (Nagoya University), John Slaney (Australian National University), Mark Steedman (University of Edinburgh), Hans Tompits (Vienna University of Technology).

The Organizing Committee members were: Makoto Kanazawa (National Institute of Informatics) (Co-chair), Anjolina G. de Oliveira (U Fed Pernambuco, Brazil), Ruy de Queiroz (U Fed Pernambuco, Brazil) (co-chair), Ken Satoh (National Institute of Informatics).

There were six invited talks:

New Characterisations of Definable Search Problems, by Arnold Beckmann (Swansea University, $\mathrm{UK})$.

Algebraic Valuations as Behavioral Logical Matrices, by Carlos Caleiro (Technical University of Lisbon, Portugal). 
Knot-Based Query Answering in Description Logic, by Thomas Eiter (Vienna University of Technology, Austria).

Recognizability in the Simply Typed Lambda-Calculus, by Sylvain Salvati (INRIA Bordeaux - Sud Ouest/LaBRI, France).

Logic-Based Probabilistic Modeling, by Taisuke Sato (Tokyo Institute of Technology, Japan).

From Mathematical Logic to Life Science Ontologies, by Frank Wolter (University of Liverpool, $\mathrm{UK})$.

The following tutorial lectures were presented (two hours each):

1. Definable Search Problems in Bounded Arithmetic, by Arnold Beckmann.

2. Reasoning Using Knots, by Thomas Eiter.

3. From Mathematical Logic to Life Science Ontologies, by Frank Wolter.

The abstracts of papers presented at the meeting have been published in the Logic Journal of the Interest Group in Pure and Applied Logics 17(6):805-815, Oxford Univ Press, Dec 2009. Volume 5514 of the series Lecture Notes in Computer Science, published by Springer, contains the proceedings of the meeting.

Reflecting the diversity of scientific investigation discussed at the meeting, the papers published in this special issue were expanded, re-refereed and chosen to appear in archival form in Fundamenta Informaticae. We should like to thank the several reviewers who anonymously contributed to keep the high standards of this journal.

The special issue editors

Hiroakira Ono

ono@jaist.ac.jp

Makoto Kanazawa

kanazawa@nii.ac.jp

Ruy de Queiroz

ruy@cin.ufpe.br 